\title{
Supercurrent and Local Coupling in the Wess-Zumino Model
}

\author{
Elisabeth $\operatorname{Kraus}^{a}$, Christian Rupp ${ }^{b}$, Klaus Sibold ${ }^{c}$, \\ ${ }^{a}$ Physikalisches Institut der Universität Bonn, \\ Nußallee 12, D - 53115 Bonn, Germany \\ Email: kraus@th.physik.uni-bonn.de \\ ${ }^{b}$ Institut für Theoretische Physik, Universität Bern, \\ Sidlerstrasse 5, CH - 3012 Bern, Switzerland \\ Email: rupp@itp.unibe.ch \\ ${ }^{c}$ Institut für Theoretische Physik, Universität Leipzig, \\ Augustusplatz 10/11, D - 04109 Leipzig, Germany \\ Email: Klaus.Sibold@itp.uni-leipzig.de
}

\begin{abstract}
We study the Wess-Zumino model with the coupling extended to a chiral superfield. In order to incorporate the renormalization effects a further external real field has to be introduced. It is then possible to derive a Callan-Symanzik equation and to prove renormalizability. By constructing the supercurrent in this context the whole machinery for describing the superconformal symmetries becomes available. The presence of the external fields allows also to define multiple insertions of all relevant composite operators. Interesting relations to the curved superspace treatment show up.
\end{abstract}

PACS numbers: 11.10.Gh, 11.30.Pb, 11.40.-q

Keywords: Supersymmetry, Renormalization, Supercurrent, Wess-Zumino Model, Local Coupling 


\section{Introduction}

The non-renormalization of chiral vertices has been a key issue in supersymmetric theories from the very first moment when one looked at the renormalization problem. At the beginning it was seen in explicit component calculations [1] and then automatically realized when performing perturbation theory in terms of superfields [2, 3, 4]. The puzzling point has always been that the supersymmetry Ward identities (WI) did not require them to hold, but the supersymmetric structure of the integrands - maintained when working with supergraphs - ensured them.

A deeper understanding has been achieved only recently [5] using two ingredients: (1) rendering the coupling local causes flow of external momentum through every internal line of a diagram and thus makes the integrand accessible to operations "from the outside"; it also opens the way to the lowest $\theta$ component of a vertex which has lower dimension than the highest one. (2) observing and exploiting the fact that an integrated vertex is always a susy variation and thus carries momentum factors. Quite a number of results have been obtained meanwhile which clearly show that this new insight is fruitful [6, 7, 8]. This refers in particular to susy gauge theories formulated in the Wess-Zumino gauge where these non-renormalization theorems would not be available in any other way than by falling over them in explicit calculations.

It is then obvious that one should study the effect of local couplings also in the context of linear realization of supersymmetry in terms of superfields and exploit the new tool. Since in the past it has become evident at many instances that the supercurrent is the carrier of the most important information on local structure in susy models we formulate in the present paper the Wess-Zumino model as an example of a chiral model with local coupling. We derive the supercurrent in presence of a local coupling and thus pave the way for the analysis of the whole superconformal properties of the model. In particular we derive a local and an integrated Callan-Symanzik equation which completes the renormalizability proof in [5] when the coupling is local.

The paper is organized as follows. In Sect. 2 we set up global WI's for two different Rsymmetries and their difference which plays a decisive role in the subsequent construction. For it gives raise to a powerful local gauge WI which in Sect. 3 is used to define the multiple insertions of the composite operator $A \bar{A}$ which contains an axial current as one of its components. It is essentially this operator which is responsible for all renormalization effects in the model. In Sect. 4 we derive the local and integrated Callan-Symanzik equation via the connection of the dilatations with scaling of parameters carrying mass dimension. In Sect. 5 we derive as an application some properties of double insertions showing thereby the usefulness of the local coupling and the external field introduced for handling the product $A \bar{A}$. The connection to the curved superspace treatment found in particular there clearly indicates that our results are as scheme independent as they can be when one is constructing currents and the like explicitly. In the conclusions we discuss our findings and give an outlook to further applications of the local coupling. 


\section{Global Ward Identities}

Introducing a local coupling in the Wess-Zumino model ( $A$ : chiral superfield)

$$
\Gamma_{\mathrm{cl}}=\frac{1}{16} \int \mathrm{d}^{8} z A \bar{A}+\int \mathrm{d}^{6} z\left(\frac{m}{8} A^{2}+\frac{\lambda}{48} A^{3}\right)+\int \mathrm{d}^{6} \bar{z}\left(\frac{m}{8} \bar{A}^{2}+\frac{\lambda}{48} \bar{A}^{3}\right)
$$

means promoting the real coupling constant $\lambda$ to chiral external fields $\Lambda, \bar{\Lambda}$ such that one constructs Feynman diagrams from

$$
\begin{aligned}
\Gamma_{\text {eff }}= & \frac{1}{16} \int \mathrm{d}^{8} z \sum_{n=0}^{\infty} z^{(n)} \hbar^{n}(\Lambda \bar{\Lambda})^{n} A \bar{A} \\
& +\int \mathrm{d}^{6} z\left(\frac{m}{8} A^{2}+\frac{1}{48} \Lambda A^{3}\right)+\int \mathrm{d}^{6} \bar{z}\left(\frac{m}{8} \bar{A}^{2}+\frac{1}{48} \bar{\Lambda} \bar{A}^{3}\right) \\
& +\frac{1}{8} \int \mathrm{d}^{8} z \sum_{n=1}^{\infty} \xi^{(n)} \hbar^{n+1}(\Lambda \bar{\Lambda})^{n}\left((\Lambda A)^{2}+(\bar{\Lambda} \bar{A})^{2}\right) .
\end{aligned}
$$

Due to the space-time dependence of $\Lambda$ and $\bar{\Lambda}$ every vertex in a diagram receives external momentum in accordance with the standard Feynman rules.

The usage of the above $\Gamma_{\text {eff }}$ refers to renormalization in the BPHZ scheme, which has been shown to be a supersymmetric invariant scheme. Hence supersymmetric Ward identities are maintained in the construction and superfield expressions can be used throughout the paper.

The terms going with $z^{(n)}, z^{(0)}=1$ describe the kinetic term and terms which reduce to its counterterms in the limit of constant coupling. Mass and interaction terms have by prescription no counterterms. This implies the non-renormalization of chiral vertices if renormalization is possible with such a choice of $\Gamma_{\text {eff }}$. The terms going with $\xi^{(n)}$ represent power counting admissible counterterms which vanish in the adiabatic limit. We have omitted possible terms which are linear in the field $A$ because they do not play a role in what follows and the omission is consistent with higher orders. Powers of $\Lambda$ and $\bar{\Lambda}$ are assigned together with orders in $\hbar$ in such a way that a $R^{\prime}$-symmetry [9, 5] is maintained naively

$$
W^{R^{\prime}} \Gamma \equiv\left(-\mathrm{i} \int \mathrm{d}^{6} z \mathrm{i}\left(-1+\theta^{\alpha} \partial_{\alpha}\right) A \frac{\delta}{\delta A}+\mathrm{i}\left(1+\theta^{\alpha} \partial_{\alpha}\right) \Lambda \frac{\delta}{\delta \Lambda}-c . c .\right) \Gamma=0
$$

The validity of this WI guarantees already the non-renormalization of chiral vertices.

An important member of the superconformal symmetry is yet another $R$-symmetry: there the fields have the so called conformal weights. For constant coupling it is always 
only softly broken. For local coupling it is again only softly broken

$$
\begin{aligned}
W^{R} \Gamma \equiv\left(-\mathrm{i} \int \mathrm{d}^{6} z \mathrm{i}\left(-\frac{2}{3}+\theta^{\alpha} \partial_{\alpha}\right) A \frac{\delta}{\delta A}+\mathrm{i} \theta^{\alpha} \partial_{\alpha} \Lambda \frac{\delta}{\delta \Lambda}-c . c .\right) \Gamma & \\
& =\frac{m}{12}\left[\int \mathrm{d}^{6} z A^{2}-\int \mathrm{d}^{6} \bar{z} \bar{A}^{2}\right]_{3} \cdot \Gamma
\end{aligned}
$$

if the $\xi^{(n)}$ are appropriately chosen, as will be shown more explicitly below. The coefficients $z^{(n)}$ can be fixed by the usual normalization condition prescribing the wave function renormalization in the flat limit. It will turn out to be useful to consider the difference of the above two $R$-symmetries which is a global $\mathrm{U}(1)$ commuting with supersymmetry:

$$
\begin{aligned}
& W^{3} \Gamma \equiv\left(W^{R^{\prime}}-W^{R}\right) \Gamma=\left(\int \mathrm{d}^{6} z\left(-\frac{1}{3} A \frac{\delta}{\delta A}+\Lambda \frac{\delta}{\delta \Lambda}\right)-\text { c.c. }\right) \Gamma \\
& W^{3} \Gamma=-\frac{m}{12}\left[\int \mathrm{d}^{6} z A^{2}-\int \mathrm{d}^{6} \bar{z} \bar{A}^{2}\right]_{3} \cdot \Gamma .
\end{aligned}
$$

One of the aims in the present paper is the derivation of the Callan-Symanzik equation (CS) which in the adiabatic limit $(\Lambda=\bar{\Lambda}=\lambda)$ is known [10, 11] to have the form

$$
\left(m \partial_{m}+2 \kappa^{2} \partial_{\kappa^{2}}+\beta \partial_{\lambda}-\gamma \mathscr{N}\right) \Gamma=\alpha \frac{m}{4}\left[\int \mathrm{d}^{6} z A^{2}+\int \mathrm{d}^{6} \bar{z} \bar{A}^{2}\right]_{3} \cdot \Gamma
$$

with

$$
\beta=3 \gamma, \quad \alpha-1=2 \gamma
$$

$\gamma$ denotes the anomalous dimension which will be given more explicitly below. $\kappa^{2}$ is the normalization point where the wave function counterterm is fixed. As long as the coupling $\Lambda$ is local one cannot express the effect of $m \partial_{m}+2 \kappa^{2} \partial_{\kappa^{2}}$ on $\Gamma$ by other differential operators and a soft mass insertion. Hence for local coupling one has to introduce another external field $L$ which is a real superfield of dimension and R-weight zero and couples accordingly; in particular it couples to the product $A \bar{A}$ and allows to absorb the hard breaking of the CS equation into a field operator (see also [6, 12]). Since by dimensional analysis

$$
\left(m \partial_{m}+2 \kappa^{2} \partial_{\kappa^{2}}\right) \Gamma=-\mathrm{i} W^{D} \Gamma
$$

where

$$
W^{D} \equiv-\mathrm{i} \int \mathrm{d}^{4} x \sum_{\phi}\left(d(\phi)+x \partial_{x}\right) \phi \frac{\delta}{\delta \phi}
$$

denotes the dilatations and furthermore a supersymmetric extension of the latter can be constructed in a systematic fashion [10, 11] we go over to local Ward identities. 


\section{Local Ward identities}

All subsequent considerations will be based on the following $\Gamma_{\text {eff }}$.

$$
\begin{aligned}
\Gamma_{\text {eff }}= & \frac{1}{16} \int \mathrm{d}^{8} z \sum_{n, m=0}^{\infty} z^{(n, m)} \hbar^{n}(\Lambda \bar{\Lambda})^{n} L^{m} A \bar{A} \\
& +\left(\int \mathrm{d}^{6} z\left(\frac{m}{8} A^{2}+\frac{1}{48} \Lambda A^{3}\right)+\text { c.c. }\right) \\
& +\frac{1}{8} \int \mathrm{d}^{8} z\left(\sum_{n, m=0}^{\infty} \xi^{(n, m)} \hbar^{n+1}(\Lambda \bar{\Lambda})^{n} L^{m}\left((\Lambda A)^{2}+(\bar{\Lambda} \bar{A})^{2}\right)\right)
\end{aligned}
$$

The systematic procedure for constructing all currents and WI's of the superconformal transformations alluded to above (chapts. 6 \& 16 in [11]) starts with contact terms

$$
\begin{aligned}
w_{\alpha}^{\mathrm{conf}}= & -\frac{2}{3} D_{\alpha}\left(A \frac{\delta}{\delta A}\right)+2 D_{\alpha} A \frac{\delta}{\delta A}+2 D_{\alpha} \Lambda \frac{\delta}{\delta \Lambda} \\
& -2 \bar{D}^{2} D_{\alpha} L \frac{\delta}{\delta L}+2 D_{\alpha} L \bar{D}^{2} \frac{\delta}{\delta L}
\end{aligned}
$$

which give rise e.g. via

$$
\hat{W}_{R}^{\text {conf }} \equiv \int \mathrm{d}^{4} x\left(D^{\alpha} w_{\alpha}^{\text {conf }}-\bar{D}_{\dot{\alpha}} \bar{w}^{\text {conf } \dot{\alpha}}\right)
$$

to the conformal $R$-symmetry contact terms and those of supersymmetry and translations

$$
\begin{aligned}
\hat{W}_{R}^{\text {conf }} & =W_{R}^{\text {conf }}+\theta^{\alpha} W_{\alpha}^{\text {susy }}+\bar{\theta}_{\dot{\alpha}} \bar{W}^{\text {susy } \dot{\alpha}}+\theta \sigma^{\nu} \bar{\theta} W_{\nu}^{\mathrm{P}} \\
W_{\alpha}^{\text {susy }} & \equiv-\mathrm{i} \int \mathrm{d}^{6} z \sum_{\phi} \delta_{\alpha}^{\text {susy }} \phi \frac{\delta}{\delta \phi} \\
W_{\nu}^{\mathrm{P}} & \equiv-\mathrm{i} \int \mathrm{d}^{6} z \sum_{\phi} \partial_{\nu} \phi \frac{\delta}{\delta \phi} \quad(\phi \in\{A, \bar{A}, \Lambda, \bar{\Lambda}, L\})
\end{aligned}
$$

Those of the $R^{\prime}$-symmetry read

$$
\begin{aligned}
w_{\alpha}^{\prime}= & -D_{\alpha}\left(A \frac{\delta}{\delta A}\right)+2 D_{\alpha} A \frac{\delta}{\delta A}+D_{\alpha}\left(\Lambda \frac{\delta}{\delta \Lambda}\right)+2 D_{\alpha} \Lambda \frac{\delta}{\delta \Lambda} \\
& -2 \bar{D}^{2} D_{\alpha} L \frac{\delta}{\delta L}+2 D_{\alpha} L \bar{D}^{2} \frac{\delta}{\delta L} .
\end{aligned}
$$

The corresponding ones for the " 3 " transformations are in a first step just the difference

$$
w_{\alpha}^{3} \equiv-\frac{1}{3} D_{\alpha}\left(A \frac{\delta}{\delta A}\right)+D_{\alpha}\left(\Lambda \frac{\delta}{\delta \Lambda}\right)
$$


But noting that they can be obtained by the application of $D_{\alpha}$ to more elementary terms and further identifying those as gauge transformations one is led to introduce

$$
\begin{aligned}
w^{3} & \equiv-\frac{1}{3} A \frac{\delta}{\delta A}+\Lambda \frac{\delta}{\delta \Lambda} \\
\tilde{w}^{3} & \equiv w^{3}+\frac{1}{3} \bar{D}^{2} \frac{\delta}{\delta L} .
\end{aligned}
$$

A quick check on

$$
\Gamma_{\mathrm{cl}}=\frac{1}{16} \int \mathrm{d}^{8} z A \mathrm{e}^{L} \bar{A}+\int \mathrm{d}^{6} z\left(\frac{m}{8} A^{2}+\frac{\lambda}{48} A^{3}\right)+\int \mathrm{d}^{6} \bar{z}\left(\frac{m}{8} \bar{A}^{2}+\frac{\lambda}{48} \bar{A}^{3}\right)
$$

yields

$$
\tilde{w}^{3} \Gamma_{\mathrm{cl}}=-\frac{m}{12} A^{2},
$$

i.e. a local WI where the current is absorbed by the inhomogeneous term of (3.10) and which is only broken by a mass term. The contact terms $\tilde{w}^{3}$ can be understood as a building block for an axial transformation, $\tilde{w}^{3}-\overline{\tilde{w}}^{3}$, but of course also for all other elements of the superconformal group which makes them extremely useful.

We shall now apply these WI operators to $\Gamma_{\text {eff }}$ and decompose the results as suggested by the supercurrent treatment [10, 11]:

$$
\begin{aligned}
-2 w_{\alpha} \Gamma & =\Delta_{\alpha} \cdot \Gamma \\
\Delta_{\alpha} \cdot \Gamma & =\bar{D}^{\dot{\alpha}} V_{\alpha \dot{\alpha}} \cdot \Gamma+2 D_{\alpha} S \cdot \Gamma-B_{\alpha} \cdot \Gamma \\
\left(D^{\alpha} w_{\alpha}-\bar{D}_{\dot{\alpha}} \bar{w}^{\dot{\alpha}}\right) \Gamma & =-\mathrm{i} \partial^{\mu} V_{\mu} \cdot \Gamma+\left(D^{2} S-\bar{D}^{2} \bar{S}\right) \cdot \Gamma
\end{aligned}
$$

where

$$
\begin{aligned}
V_{\mu} & \equiv \sigma_{\mu}^{\alpha \dot{\alpha}} V_{\alpha \dot{\alpha}} \\
D^{\alpha} B_{\alpha}-\bar{D}_{\dot{\alpha}} \bar{B}^{\dot{\alpha}} & =0 .
\end{aligned}
$$

The decomposition (3.14) into current, $S$-type and $B$-type breaking represents the most general situation. If a mass term is present the best one can achieve is a soft mass term plus breaking of $S$-type which means that all superconformal anomalies can be represented in terms of $S$. The supercurrent contains in this case a current for a softly broken $R$-symmetry, strictly conserved supersymmetry currents and a conserved energymomentum tensor. In the massless case one can have the $B$-type breaking which implies that all components of the supercurrent are strictly conserved currents and the breaking of superconrformal symmetry is described in terms of a real multiplet $B$. In the present case we let mimic the $R^{\prime}$-supercurrent the situation with $B$-type breaking because the mass term is $R^{\prime}$-invariant. The $S$-type breaking is represented by the "conformal" contact terms, current and breaking, whereas the "3" interpolates between the two and will contain both types of breaking. Parenthetically we remark that in the curved superspace 
treatment this assignment arises automatically.

Switching back and forth between $R^{\prime}$ and $R^{\text {conf }}$ and using at appropriate places the information originating from $\tilde{w}^{3}$ we shall be able to derive first a local and then a global CS equation. These calculations become more involved than in the classical approximation because in higher orders the breaking going with the mass should be soft, hence one needs first of all a Zimmermann identity relating the oversubtracted mass term to the minimally subtracted one. It reads

$$
\begin{aligned}
{\left[m A^{2}\right]_{3} \cdot \Gamma=} & {\left[m A^{2}\right]_{2} \cdot \Gamma } \\
& +\sum_{n=1, m=0}^{\infty} \hbar^{n}\left[u_{\text {kin }}^{(n, m)} L_{\text {kin }}^{(n, m)}+u_{\xi}^{(n-1, m)} L_{\xi}^{(n-1, m)}+u_{\xi}^{\prime(n-1, m)} L_{\xi}^{\prime(n-1, m)}\right]_{3} \cdot \Gamma \\
& +\Delta_{\text {triv }}
\end{aligned}
$$

with

$$
\begin{aligned}
L_{\text {kin }}^{(n, m)} & =\bar{D}^{2}\left((\Lambda \bar{\Lambda})^{n} L^{m} A \bar{A}\right) \\
L_{\xi}^{(n, m)} & =\bar{D}^{2}\left((\Lambda \bar{\Lambda})^{n} L^{m}(\Lambda A)^{2}\right) \\
L_{\xi}^{\prime(n, m)} & =\bar{D}^{2}\left((\Lambda \bar{\Lambda})^{n} L^{m}(\bar{\Lambda} \bar{A})^{2}\right)
\end{aligned}
$$

Here, $\Delta_{\text {triv }}$ contains all terms with zero or one propagating fields. These are trivial in the sense that they cannot contribute to 1PI loop diagrams in the adiabatic limit and do not affect the discussion of the non-trivial breaking terms. In this paper we do not consider $\Delta_{\text {triv }}$, however one should keep in mind that these terms contribute to Ward identities if tested w.r.t less than two dynamical fields.

Our first aim is the local gauge WI

$$
\begin{aligned}
\tilde{w}^{3} \Gamma= & {\left[\tilde{w}^{3} \Gamma_{\text {eff }}\right]_{3} \cdot \Gamma } \\
\tilde{w}^{3} \Gamma= & -\frac{1}{12} m\left[A^{2}\right]_{2} \cdot \Gamma \\
& +\left[\frac{1}{16} \sum_{n, m=0}^{\infty} z^{(n, m)} \hbar^{n}\left\{\left(n-\frac{1}{3}\right) L_{\text {kin }}^{(n, m)}+\frac{m}{3} L_{\text {kin }}^{(n, m-1)}\right\}\right. \\
& \left.\quad-\frac{1}{12} \sum_{n=1, m=0}^{\infty} u_{\text {kin }}^{(n, m)} \hbar^{n} L_{\text {kin }}^{(n, m)}\right]_{3} \cdot \Gamma \\
+ & {\left[\frac{1}{8} \sum_{n, m=0}^{\infty} \xi^{(n, m)} \hbar^{n+1}\left\{\left(n+\frac{4}{3}\right) L_{\xi}^{(n, m)}+\frac{m}{3} L_{\xi}^{(n, m-1)}+n L_{\xi}^{\prime(n, m)}+\frac{m}{3} L_{\xi}^{\prime(n, m-1)}\right\}\right.} \\
& \left.\quad-\frac{1}{12} \sum_{n=0, m=0}^{\infty} \hbar^{n+1}\left(u_{\xi}^{(n, m)} L_{\xi}^{(n, m)}+u_{\xi}^{\prime(n, m)} L_{\xi}^{\prime(n, m)}\right)\right]_{3} \cdot \Gamma
\end{aligned}
$$

We would like to dispose over the arbitrary coefficients $z^{(n, m)}$ and $\xi^{(n, m)}$ in such a way that the r.h.s. of (3.23) reduces to the soft term. If this is possible we are closest to the 
classical situation as expressed by (3.12). We have to solve the equations

$$
\begin{aligned}
\frac{3}{4}\left(n-\frac{1}{3}\right) z^{(n, m)}+\frac{1}{4}(m+1) z^{(n, m+1)}-u_{\text {kin }}^{(n, m)} & =0 \\
\frac{3}{2}\left(n+\frac{4}{3}\right) \xi^{(n, m)}+\frac{1}{2}(m+1) \xi^{(n, m+1)}-u_{\xi}^{(n, m)} & =0 \\
\frac{3}{2} n \xi^{(n, m)}+\frac{1}{2}(m+1) \xi^{(n, m+1)}-u_{\xi}^{(n, m)} & =0
\end{aligned}
$$

(3.24) with $z^{(0,0)}=1$ is readily solved by

$$
\begin{aligned}
z^{(0, m)} & =\frac{1}{m !} \\
z^{(n, 0)} & =\text { arbitrary for } n \geq 1 \\
(m+1) z^{(n, m+1)} & =(1-3 n) z^{(n, m)}+4 u_{\text {kin }}^{(n, m)} \text { for } n \geq 1, m \geq 0
\end{aligned}
$$

The difference of $(3.26)$ and $(3.25)$ determines

$$
\xi^{(n, m)}=\frac{1}{2}\left(u_{\xi}^{(n, m)}-u_{\xi}^{\prime(n, m)}\right)
$$

with $\xi^{(0,0)}=0$.

In order to show that (3.26), (3.25) hold also separately, we consider the consistency condition

$$
\left[\tilde{w}^{3}(z), \overline{\tilde{w}}^{3}\left(z^{\prime}\right)\right]=0
$$

which leads to

$$
\left(n+\frac{4}{3}\right) u_{\xi}^{\prime(n, m)}+\frac{1}{3}(m+1) u_{\xi}^{\prime(n, m+1)}=n u_{\xi}^{(n, m)}+\frac{1}{3}(m+1) u_{\xi}^{(n, m+1)} .
$$

By inserting (3.32) into (3.30), one finds that both (3.26) and (3.25) are satisfied.

Thus we have

$$
\tilde{w}^{3} \Gamma=\left(w^{3}+\frac{1}{3} \bar{D}^{2} \frac{\delta}{\delta L}\right) \Gamma=-\frac{1}{12} m\left[A^{2}\right]_{2} \cdot \Gamma+Q_{\text {triv }}^{3}
$$

i.e. also on the quantized level we have a gauge theory with abelian gauge invariance which is only broken a soft mass term. With (3.27) the respective classical action has the form

$$
\Gamma_{\mathrm{cl}}=\frac{1}{16} \int \mathrm{d}^{8} z A \mathrm{e}^{L} \bar{A}+\int \mathrm{d}^{6} z\left(\frac{m}{8} A^{2}+\frac{\lambda}{48} A^{3}\right)+\int \mathrm{d}^{6} \bar{z}\left(\frac{m}{8} \bar{A}^{2}+\frac{\lambda}{48} \bar{A}^{3}\right)
$$

The result (3.33) can be brought very simply into a form where the machinery of forming moments is applicable and thus the complete superconformal structure becomes available. 
We rewrite (3.33) as a trace equation.

$$
\begin{aligned}
w_{\alpha}^{3} & \equiv D_{\alpha} w^{3}=-\frac{1}{3} D_{\alpha} \bar{D}^{2} \frac{\delta \Gamma}{\delta L}-\frac{1}{12}\left[m D_{\alpha} A^{2}\right]_{5 / 2} \cdot \Gamma \\
-2 w_{\alpha}^{3} \Gamma & =\bar{D}^{\dot{\alpha}} V_{\alpha \dot{\alpha}}^{3} \cdot \Gamma+2 D_{\alpha} S^{3} \cdot \Gamma-B_{\alpha}^{3} \cdot \Gamma \\
V_{\alpha \dot{\alpha}}^{3} \equiv & \frac{4}{3}\left[D_{\alpha}, \bar{D}_{\dot{\alpha}}\right] \frac{\delta \Gamma_{\mathrm{eff}}}{\delta L} \\
S^{3} \equiv & -\frac{1}{12} m A^{2} \\
B_{\alpha}^{3} \equiv & 2 \bar{D}^{2} D_{\alpha} \frac{\delta \Gamma_{\mathrm{eff}}}{\delta L} \\
\frac{\delta \Gamma_{\mathrm{eff}}}{\delta L}= & \frac{1}{16} \sum_{n=0, m=1}^{\infty} z^{(n, m)} \hbar^{n} m L^{m-1}(\Lambda \bar{\Lambda})^{n} A \bar{A} \\
& +\frac{1}{8} \sum_{n=0, m=1}^{\infty} \xi^{(n, m)} \hbar^{n+1} m L^{m-1}(\Lambda \bar{\Lambda})^{n}\left(\Lambda^{2} A^{2}+\bar{\Lambda}^{2} \bar{A}^{2}\right)
\end{aligned}
$$

The next aim is now to derive the trace equation for the conformal contact terms. Together with (3.36) it will contain all information on the model. For the supercurrent generated by the conformal $R$-symmetry we find

$$
\begin{aligned}
V_{\alpha \dot{\alpha}}^{\text {conf }}=-\frac{1}{6} \sum_{n, m} z^{(n, m)} \hbar^{n} & \left(L^{m}\left(D_{\alpha}\left(\Lambda^{n} A\right) \bar{D}_{\dot{\alpha}}\left(\bar{\Lambda}^{n} \bar{A}\right)-\Lambda^{n} A D_{\alpha} \bar{D}_{\dot{\alpha}}\left(\bar{\Lambda}^{n} \bar{A}\right)+\bar{D}_{\dot{\alpha}} D_{\alpha}\left(\Lambda^{n} A\right) \bar{\Lambda}^{n} \bar{A}\right)\right. \\
& +D_{\alpha} L^{m} \Lambda^{n} A \bar{D}_{\dot{\alpha}}\left(\bar{\Lambda}^{n} \bar{A}\right)-\bar{D}_{\dot{\alpha}} L^{m} D_{\alpha}\left(\Lambda^{n} A\right) \bar{\Lambda}^{n} \bar{A} \\
& \left.+\Lambda^{n} A \bar{\Lambda}^{n} \bar{A}\left(-m L^{m-1}\left[D_{\alpha}, \bar{D}_{\dot{\alpha}}\right] L+m(m-1) L^{m-2} D_{\alpha} L \bar{D}_{\dot{\alpha}} L\right)\right) \\
-\frac{1}{3} \sum_{n, m} \xi^{(n, m)} \hbar^{n+1} & \left(L ^ { m } \left(D_{\alpha}\left(\Lambda^{n+2} A^{2}\right) \bar{D}_{\dot{\alpha}}\left(\bar{\Lambda}^{n}\right)\right.\right. \\
& \left.-\Lambda^{n+2} A^{2} D_{\alpha} \bar{D}_{\dot{\alpha}}\left(\bar{\Lambda}^{n}\right)+\bar{D}_{\dot{\alpha}} D_{\alpha}\left(\Lambda^{n+2} A^{2}\right) \bar{\Lambda}^{n}\right) \\
& +D_{\alpha} L^{m} \Lambda^{n+2} A^{2} \bar{D}_{\dot{\alpha}}\left(\bar{\Lambda}^{n}\right)-\bar{D}_{\dot{\alpha}} L^{m} D_{\alpha}\left(\Lambda^{n+2} A^{2}\right) \bar{\Lambda}^{n} \\
& +\Lambda^{n+2} A^{2} \bar{\Lambda}^{n}\left(-m L^{m-1}\left[D_{\alpha}, \bar{D}_{\dot{\alpha}}\right] L+m(m-1) L^{m-2} D_{\alpha} L \bar{D}_{\dot{\alpha}} L\right) \\
& + \text { c.c. })
\end{aligned}
$$

It is instructive to extract the classical approximation

$$
\begin{aligned}
V_{\alpha \dot{\alpha}}^{\text {conf,class }}=-\frac{1}{6} \mathrm{e}^{L}\left(D_{\alpha} A\right. & \bar{D}_{\dot{\alpha}} \bar{A}-A D_{\alpha} \bar{D}_{\dot{\alpha}} \bar{A}+\bar{D}_{\dot{\alpha}} D_{\alpha} A \bar{A} \\
& +D_{\alpha} L A \bar{D}_{\dot{\alpha}} \bar{A}-\bar{D}_{\dot{\alpha}} L D_{\alpha} A \bar{A} \\
& \left.-\left[D_{\alpha}, \bar{D}_{\dot{\alpha}}\right] L A \bar{A}+D_{\alpha} L \bar{D}_{\dot{\alpha}} L A \bar{A}\right)
\end{aligned}
$$

and also to present the limit $L=0, \Lambda=\bar{\Lambda}=\lambda=$ const.

$$
\begin{aligned}
V_{\alpha \dot{\alpha}}^{\lim }= & -\frac{1}{6} z\left(D_{\alpha} A \bar{D}_{\dot{\alpha}} \bar{A}-A D_{\alpha} \bar{D}_{\dot{\alpha}} \bar{A}+\bar{D}_{\dot{\alpha}} D_{\alpha} A \bar{A}\right) \\
& -\frac{1}{3} \xi\left\{D_{\alpha}, \bar{D}_{\dot{\alpha}}\right\}\left(A^{2}-\bar{A}^{2}\right)
\end{aligned}
$$


with

$$
\begin{aligned}
z & =\sum_{n} z^{(n, 0)} \hbar^{n} \lambda^{2 n} \\
\xi & =\sum_{n} \xi^{(n, 0)} \hbar^{n+1} \lambda^{2 n+2} \\
\xi^{(n, 0)} & =\frac{1}{2}\left(u_{\xi}^{(n, 0)}-u_{\xi}^{\prime(n, 0)}\right)
\end{aligned}
$$

We see that our construction yields a $\xi$ contribution to the supercurrent precisely as the curved superspace treatment [13] does. Hence local coupling resolves the decomposition ambiguity $V \leftrightarrow S$ for these terms as the invariance requirement with respect to diffeomorphism.

The other contributions to the trace equation

$$
-2 w_{\alpha}^{\mathrm{conf}}=\bar{D}^{\dot{\alpha}} V_{\alpha \dot{\alpha}}^{\mathrm{conf}} \cdot \Gamma+2 D_{\alpha} S^{\mathrm{conf}} \cdot \Gamma-B_{\alpha}^{\mathrm{conf}} \cdot \Gamma
$$

turn out to be

$$
\begin{aligned}
S^{\mathrm{conf}}= & -\frac{1}{12} m\left[A^{2}\right]_{2} \\
& -\frac{1}{12}\left[\sum_{n, m} u_{\text {kin }}^{(n, m)} \hbar^{n} L_{\text {kin }}^{(n, m)}+\sum_{n, m} \frac{1}{2}\left(u_{\xi}^{(n, m)}+u_{\xi}^{\prime(n, m)}\right) \hbar^{n+1}\left(L_{\xi}^{(n, m)}+L_{\xi}^{\prime(n, m)}\right)\right]_{3} \\
B_{\alpha}^{\mathrm{conf}}= & 0 .
\end{aligned}
$$

\section{The CS equation: local and integrated}

The trace equation (3.47) leads via the moment construction to the local dilatation WI

$$
\begin{aligned}
& W^{D} \Gamma=\left.\hat{W}^{D} \Gamma\right|_{\tilde{\theta}=0} \\
& \hat{W}^{D}= \int \mathrm{d}^{4} x w^{D} \\
& w^{D}= w_{\text {trace }}^{P}+x^{\nu} w_{\nu}^{P} \\
& w_{\text {trace }}^{P}= \frac{3}{2} \mathrm{i}\left(D^{\alpha} w_{\alpha}+\bar{D}_{\dot{\alpha}} \bar{w}^{\dot{\alpha}}\right) \\
& w_{\nu}^{P}= \frac{1}{16}\left(-\left(D^{2} \bar{D}_{\dot{\alpha}} w_{\alpha}+\bar{D}^{2} D_{\alpha} \bar{w}_{\dot{\alpha}}\right) \sigma_{\nu}^{\alpha \dot{\alpha}}-\sigma_{\nu}^{\beta \dot{\beta}}\left\{D_{\beta}, \bar{D}_{\dot{\beta}}\right\}\left(D^{\alpha} w_{\alpha}-\bar{D}_{\dot{\alpha}} \bar{w}^{\dot{\alpha}}\right)\right. \\
&\left.\quad+8 \mathrm{i} \partial_{\nu}\left(D^{\alpha} w_{\alpha}+\bar{D}_{\dot{\alpha}} \bar{w}^{\dot{\alpha}}\right)\right) \\
& w^{D} \Gamma=\partial^{\mu} \hat{D}_{\mu} \cdot \Gamma-\frac{3}{2} \mathrm{i}\left(D^{2} S^{\operatorname{conf}}+\bar{D}^{2} \bar{S}^{\text {conf }}\right) \cdot \Gamma
\end{aligned}
$$

A local CS equation is given by the equation

$$
w_{\text {trace }}^{P} \Gamma \equiv \frac{3}{2} \mathrm{i}\left(D^{\alpha} w_{\alpha}+\bar{D}_{\dot{\alpha}} \bar{w}^{\dot{\alpha}}\right) \Gamma=-\frac{3}{2} \mathrm{i}\left(D^{2} S^{\text {conf }}+\bar{D}^{2} \bar{S}^{\text {conf }}\right) \cdot \Gamma
$$


(which incidentally explains the origin of the name "trace equation" for (3.47)).

It is now crucial to observe that the hard terms in $S^{\text {conf }}$ can be represented by a local functional operator which commutes with $\tilde{w}^{3}$

$$
\mathscr{B}_{\text {loc }} \equiv \frac{1}{2} \sum_{r=1}^{\infty} \gamma^{(r)} \hbar^{r} \bar{D}^{2}\left\{(\Lambda \bar{\Lambda})^{r} \mathrm{e}^{-3 r L} \frac{\delta}{\delta L}\right\}
$$

acting on $\Gamma$, i.e.

$$
\left(w_{\text {trace }}^{P}+\mathrm{i} D^{2} \mathscr{B}_{\text {loc }}+\mathrm{i} \bar{D}^{2} \overline{\mathscr{B}}_{\text {loc }}\right) \Gamma=\frac{\mathrm{i}}{8} m\left[D^{2} A^{2}+\bar{D}^{2} \bar{A}^{2}\right]_{3} \cdot \Gamma
$$

In order to prove this we first calculate

$$
\begin{aligned}
\mathscr{B}_{\mathrm{loc}} \Gamma_{\mathrm{eff}}= & \sum_{n, m} \sum_{r=1}^{n} \sum_{s=0}^{m} \frac{1}{32} \frac{(-3 r)^{s}}{s !} \gamma^{(r)} z^{(n-r, m+1-s)}(m+1-s) \hbar^{n} L_{\mathrm{kin}}^{(n, m)} \\
& +\sum_{n, m} \sum_{r=1}^{n} \sum_{s=0}^{m} \frac{1}{16} \frac{(-3 r)^{s}}{s !} \gamma^{(r)} \xi^{(n-r, m+1-s)}(m+1-s) \hbar^{n}\left(L_{\xi}^{(n, m)}+L_{\xi}^{\prime(n, m)}\right)
\end{aligned}
$$

This coincides with the hard terms in $S^{\text {conf }}$ if

$$
\begin{array}{ll}
0=u_{\text {kin }}^{(n, m)}+\frac{1}{4} \sum_{r=1}^{n} \sum_{s=0}^{m} \frac{(-3 r)^{s}}{s !} \gamma^{(r)} z^{(n-r, m+1-s)}(m+1-s) & \equiv q_{1}(n, m) \\
0=u_{\xi}^{(n, m)}+u_{\xi}^{(n, m)}+\sum_{r=1}^{n} \sum_{s=0}^{m} \frac{(-3 r)^{s}}{s !} \gamma^{(r)} \xi^{(n-r, m+1-s)}(m+1-s) & \equiv q_{2}(n, m) .
\end{array}
$$

By properly adjusting $\gamma^{(r)}, q_{1}(n, 0)$ can be made to vanish,

$$
\begin{aligned}
& \gamma^{(1)}=-4 u_{\mathrm{kin}}^{(1,0)} \\
& \gamma^{(n)}=-4 u_{\mathrm{kin}}^{(n, 0)}-\sum_{r=1}^{n-1} \gamma^{(r)} z^{(n-r, 1)} \quad(n \geq 2)
\end{aligned}
$$

Before we show that the remaining $q_{1}, q_{2}$ vanish automatically, we pass over from (4.9) to the integrated CS equation.

$$
W^{D} \Gamma+\mathrm{i} \mathscr{B} \Gamma=\frac{\mathrm{i}}{8} m\left[\int \mathrm{d}^{6} z A^{2}+\int \mathrm{d}^{6} \bar{z} \bar{A}^{2}\right]_{3} \cdot \Gamma,
$$

where $\mathscr{B}$ is the integrated version of $\mathscr{B}_{\text {loc }}$,

$$
\mathscr{B}=\int \mathrm{d}^{6} z \mathscr{B}_{\text {loc }}+\int \mathrm{d}^{6} \bar{z} \overline{\mathscr{B}}_{\text {loc }}
$$


Thus the integrated CS equation reads

$$
\mathscr{C} \Gamma=\frac{1}{8} m\left[\int \mathrm{d}^{6} z A^{2}+\int \mathrm{d}^{6} \bar{z} \bar{A}^{2}\right]_{3} \cdot \Gamma
$$

with the CS operator

$$
\mathscr{C}=m \partial_{m}+2 \kappa^{2} \partial_{\kappa^{2}}+\mathscr{B} .
$$

In order to finally prove (4.12), we consider the commutator

$$
\left[\mathscr{C}, \tilde{w}^{3}(z)\right]=0
$$

Since $\tilde{w}^{3} \Gamma=$ soft, this implies

$$
\tilde{w}^{3}(z) \mathscr{C} \Gamma=\text { soft }
$$

For the lowest non-vanishing order $n$ this means

$$
\begin{aligned}
q_{1}(n, m+1) & =\frac{3 n-1}{m+1} q_{1}(n, m) \\
q_{2}(n, m) & =0 .
\end{aligned}
$$

Since $q_{1}(n, 0)=0$ due to our choice of $\gamma^{(r)}$, this shows that (4.11) and (4.12) hold, and we have thus established the local and integrated CS equations (4.9), (4.17). Moreover, the trace equation (3.47) may now be nicely expressed as

$$
-2 w_{\alpha}^{\mathrm{conf}}=\bar{D}^{\dot{\alpha}} V_{\alpha \dot{\alpha}}^{\mathrm{conf}} \cdot \Gamma+\frac{4}{3} D_{\alpha} \mathscr{B}_{\mathrm{loc}} \Gamma-\frac{1}{6} m\left[D_{\alpha} A^{2}\right]_{5 / 2} \cdot \Gamma .
$$

In order to make contact with the standard Wess-Zumino model, we take the limit $L=0, \Lambda=\bar{\Lambda}=\lambda=$ const. First we define

$$
\begin{aligned}
z & =\sum_{n} z^{(n, 0)} \hbar^{n} \lambda^{2 n} & \xi & =\sum_{n} \xi^{(n, 0)} \hbar^{n+1} \lambda^{2 n+2} \\
\gamma & =-\frac{1}{2} \sum_{r} \gamma^{(r)} \hbar^{r} \lambda^{2 r} & \beta & =3 \lambda \gamma \\
\tilde{z} & =\sum_{n} z^{(n, 1)} \hbar^{n} \lambda^{2 n} & u_{\text {kin }} & =\sum_{n} u_{\text {kin }}^{(n, 0)} \hbar^{n} \lambda^{2 n}
\end{aligned}
$$

From (4.11), (3.29) we know that

$$
\gamma=\frac{2 u_{\text {kin }}}{\tilde{z}}, \quad \tilde{z}=\left(1-\frac{3}{2} \lambda \partial_{\lambda}\right) z+4 u_{\text {kin }},
$$

such that the standard form for $\gamma$ is recovered,

$$
\gamma=\frac{2 u_{\text {kin }}}{z+4 u_{\text {kin }}-\frac{3}{2} \lambda \partial_{\lambda} z} .
$$


In the considered limit, the operator $\mathscr{B}_{\text {loc }}$ takes a simple form,

$$
\mathscr{B}_{\text {loc }} \rightarrow-\gamma \bar{D}^{2} \frac{\delta}{\delta L}
$$

Taking into account the gauge WI (3.33), we find

$$
\begin{aligned}
\mathscr{B}_{\mathrm{loc}} \Gamma \rightarrow-\gamma A \frac{\delta}{\delta A} \Gamma+\beta \frac{\delta}{\delta \Lambda} \Gamma+\frac{1}{4} \gamma m\left[A^{2}\right]_{2} \cdot \Gamma \\
\mathscr{B} \Gamma \rightarrow\left(-\gamma \mathscr{N}_{A}+\beta \partial_{\lambda}\right) \Gamma+\frac{1}{4} \gamma m\left[\int \mathrm{d}^{6} z A^{2}+\int \mathrm{d}^{6} z \bar{A}^{2}\right]_{3} \cdot \Gamma,
\end{aligned}
$$

which reproduces the usual CS equation (2.7). The crucial point is that in the WessZumino model with local coupling a Callan-Symanzik equation can be formulated only with the help of an additional external field, but in the limit of constant coupling this

field couples just to the coupling constant and wave function renormalization operators. In our approach, the relation (4.25) between the $\beta$ and $\gamma$ functions is a consequence of the gauge WI (3.33).

It is furthermore interesting to note that $\frac{\delta}{\delta \Lambda} \Gamma_{\text {eff }}$ may be interpreted as the $\lambda$-derivative of a Lagrangian density,

$$
\begin{aligned}
\left.\frac{\delta \Gamma_{\mathrm{eff}}}{\delta \Lambda}\right|_{\substack{L=0 \\
\Lambda=\lambda}} & =\partial_{\lambda} L_{\mathrm{eff}} \\
\left.\Gamma_{\mathrm{eff}}\right|_{\substack{L=0 \\
\Lambda=\lambda}} & =\int \mathrm{d}^{6} z L_{\mathrm{eff}}+\int \mathrm{d}^{6} \bar{z} \bar{L}_{\mathrm{eff}} \\
L_{\mathrm{eff}} & \equiv \frac{1}{32} z \bar{D}^{2}(A \bar{A})+\frac{1}{48} \lambda A^{3}+\frac{1}{8} \varepsilon \bar{D}^{2} \bar{A}^{2} \\
\varepsilon & \equiv \sum_{n} \xi^{(n, 0)} \hbar^{n+1} \frac{n}{2 n+2} \lambda^{2 n+2},
\end{aligned}
$$

such that 4.23 ) reduces to

$$
-2 w_{\alpha}^{(\gamma)} \Gamma=\bar{D}^{\dot{\alpha}} V_{\alpha \dot{\alpha}} \cdot \Gamma+\frac{4}{3} D_{\alpha}\left(\beta \partial_{\lambda} L_{\mathrm{eff}}\right) \cdot \Gamma-\frac{1}{6}(1-2 \gamma) m D_{\alpha}\left[A^{2}\right]_{2} \cdot \Gamma
$$

with the anomalous contact terms

$$
w_{\alpha}^{(\gamma)} \equiv w_{\alpha}^{\mathrm{conf}}-\frac{2}{3} \gamma D_{\alpha}\left(A \frac{\delta}{\delta A}\right)
$$

This result coincides with the flat space limit of the construction of 13 .

\section{Application}

The main reason to introduce a local coupling constant is that it gives some new insight into the non-renormalization theorems of chiral vertices [5]. The present paper extends 
the considerations of [5] in supplying the Callan-Symanzik equations established in the previous section and thus completing the proof of renormalizability for the model under consideration. However, the external fields $\Lambda$ and $L$ may also be used to generate insertions of local operators,

$$
\begin{aligned}
& \left.\Delta_{L} \equiv \frac{\delta \Gamma_{\text {eff }}}{\delta L}\right|_{L=0, \Lambda=\lambda}=\frac{1}{16} A \bar{A}+O(\hbar) \\
& \left.\Delta_{\lambda} \equiv \frac{\delta \Gamma_{\text {eff }}}{\delta \Lambda}\right|_{L=0, \Lambda=\lambda}=\frac{1}{48} A^{3}+O(\hbar) .
\end{aligned}
$$

Since we are working with off-shell Ward identities, we also have access to the covariance properties of these operator insertions. For example, we find for the dilatational transformation of the $\Delta_{L}$-insertion:

$$
W^{D}\left(\left[\Delta_{L}\right] \cdot \Gamma\right) \sim\left[\delta^{D} \Delta_{L}\right] \cdot \Gamma+\left(-\gamma \mathscr{N}_{A}+\beta \partial_{\lambda}+3 \lambda \partial_{\lambda} \gamma\right)\left(\left[\Delta_{L}\right] \cdot \Gamma\right)
$$

with

$$
\delta^{D} \Delta_{L}=\left(x^{\mu} \partial_{\mu}+\frac{1}{2} \theta^{\alpha} \partial_{\alpha}-\frac{1}{2} \bar{\theta}_{\dot{\alpha}} \bar{\partial}^{\dot{\alpha}}+2\right) \Delta_{L}
$$

Here, $W^{D}$ represents only the transformation of the fields $A, \bar{A} . \sim$ means equality up to soft terms. Since $\Delta_{L}$ contains two dynamical fields, one might naively expect its anomalous dimension to be $2 \gamma$. Instead we find that its anomalous dimension is given by $3 \lambda \partial_{\lambda} \gamma$

The scaling behavior of $\Delta_{\lambda}$ is given by

$$
\begin{aligned}
W^{D}\left(\left[\Delta_{\lambda}\right] \cdot \Gamma\right) & \sim\left[\delta^{D} \Delta_{\lambda}\right] \cdot \Gamma+\left(-\gamma \mathscr{N}_{A}+\beta \partial_{\lambda}+3 \gamma\right)\left(\left[\Delta_{\lambda}\right] \cdot \Gamma\right)-\lambda \partial_{\lambda} \gamma\left[\bar{D}^{2} \Delta_{L}\right] \cdot \Gamma \\
\delta^{D} \Delta_{\lambda} & =\left(x^{\mu} \partial_{\mu}+\frac{1}{2} \theta^{\alpha} \partial_{\alpha}-\frac{1}{2} \bar{\theta}_{\dot{\alpha}} \bar{\partial}^{\dot{\alpha}}+3\right) \Delta_{\lambda}
\end{aligned}
$$

Hence this operator indeed has the naive anomalous dimension $3 \gamma$, but dilatations do not close on $\Delta_{\lambda}$ alone: the operator $\Delta_{L}$ is also involved here.

One can also produce multiple insertions of $\Delta_{\lambda}, \Delta_{L}$. For example, it follows from (3.33) by differentiating w.r.t. $L$ and then taking the adiabatic limit that

$$
\lambda\left\langle\left\{\Delta_{\lambda}\left(z_{1}\right) \cdot \Delta_{L}\left(z_{2}\right)\right\}\right\rangle \sim-\frac{1}{3}\left\langle\left\{\bar{D}^{2} \Delta_{L}\left(z_{1}\right) \cdot \Delta_{L}\left(z_{2}\right)\right\}\right\rangle+u_{\text {geom }} \bar{D}^{2} \square \delta^{8}\left(z_{1}-z_{2}\right) .
$$

The last term represents the only possible contribution from $\Delta_{\text {triv }}$ (3.18). $u_{\text {geom }}$ is a power series in $\lambda$ and $\hbar$. For the definition of double insertions, see [14. Differentiation of (3.33) w.r.t. $\Lambda$ yields

$$
\lambda\left\langle\left\{\Delta_{\lambda}\left(z_{1}\right) \cdot \Delta_{\lambda}\left(z_{2}\right)\right\}\right\rangle \sim-\frac{1}{3}\left\langle\left\{\bar{D}^{2} \Delta_{L}\left(z_{1}\right) \cdot \Delta_{\lambda}\left(z_{2}\right)\right\}\right\rangle,
$$

this time $\Delta_{\text {triv }}$ does not contribute. 


\section{Conclusions}

We have constructed the Wess-Zumino model with local coupling represented by a chiral external field $\Lambda$ and derived the supercurrent in its presence. A further necessary ingredient was an external real superfield $L$ coupled to the composite operator $A \bar{A}$. From a merely technical point of view it is this composite operator which causes all renormalizations in the model once one has encoded the fact that chiral vertices are not renormalized. The wavefunction renormalization and subsequently the anomalous dimension of the chiral field $A$ as well as the $\beta$-function of the model can all be related to the effect of inserting $A \bar{A}$ : certainly the most clear cut implementation of the non-renormalization of chiral vertices and its consequences. Speaking in equations the consequences are expressed by the local CS equation which was derived in the context of the supercurrent with its moment construction giving access to the entire superconformal group. Like in the other examples of the local coupling approach it is an axial current and its WI which plays the most decisive role in the derivation of these results: the respective local WI leads one to the interrelations of all potential anomaly coefficients. The local coupling and the external field $L$ also serve the purpose of defining multiple insertions of the respective composite operators $A^{3}$ and $A \bar{A}$ including their transformations under the superconformal group. It is also interesting to observe that the local coupling approach yields some results which had been obtained previously by going to the curved superspace. Hence there is a connection which deserves further study. This comment also applies to the study of multiple insertions to which we hope to come back in the near future. Summarizing we may say that the introduction of a local coupling is without any doubt the key to understand all renormalization questions in the Wess-Zumino model.

\section{References}

[1] J. Wess and B. Zumino, Phys. Lett. B49 (1974) 52.

[2] K. Fujikawa and W. Lang, Nucl. Phys. B88 (1975) 61.

[3] S. Ferrara and O. Piguet, Nucl. Phys. B93 (1975) 261.

[4] M.T. Grisaru, W. Siegel and M. Rocek, Nucl. Phys. B159 (1979) 429; M.T. Grisaru and W. Siegel, Nucl. Phys. B201 (1982) 292.

[5] R. Flume and E. Kraus, Nucl. Phys. B569 (2000) 625 arXiv:hep-th/9907120.

[6] E. Kraus and D. Stöckinger, Nucl. Phys. B626 (2002) 73 [arXiv:hep-th/0105028].

[7] E. Kraus, Nucl. Phys. B620 (2002) 55 arXiv:hep-th/0107239].

[8] E. Kraus, hep-ph/0110323. 
[9] N. Seiberg, Phys. Lett. B318 (1993) 469 arXiv:hep-ph/9309335.

[10] T. E. Clark, O. Piguet and K. Sibold, Nucl. Phys. B143 (1978) 445.

[11] O. Piguet and K. Sibold, "Renormalized Supersymmetry. The Perturbation Theory Of N=1 Supersymmetric Theories In Flat Space-Time," Boston, USA: Birkhäuser (1986) 346 P. ( Progress In Physics, 12).

[12] N. Arkani-Hamed, G.F. Giudice, M.A. Luty and R.Rattazzi, Phys. Rev. D58 (1998) 115005 arXiv:hep-ph/9803290.

[13] J. Erdmenger, C. Rupp and K. Sibold, Nucl. Phys. B530 (1998) 501 arXiv:hepth/9804053].

[14] J. Erdmenger and C. Rupp, Annals Phys. 276 (1999) 152 arXiv:hep-th/9811209. 